\title{
ASO Visual Abstract: Cumulative Burden of Postoperative Complications in Patients Undergoing Surgery for Primary Retroperitoneal Sarcoma
}

Fabio Tirotta, MD ${ }^{1}$ (1) , Alessandro Parente, MD $^{1}$, James Hodson, $\mathbf{B S c}^{2}$, Anant Desai, FRCS, MD $^{1}$, L. Max Almond, FRCS, MD ${ }^{1}$, and Samuel J. Ford, FRCS, PhD ${ }^{1}$

${ }^{1}$ Department of Sarcoma and General Surgery, Midlands Abdominal and Retroperitoneal Sarcoma Unit, University Hospital Birmingham NHS Foundation Trust, Birmingham, UK; ${ }^{2}$ Department of Medical Statistics, Institute of Translational Medicine, University Hospital Birmingham NHS Foundation Trust, Birmingham, UK

An increased burden of postoperative complications after excision of primary retroperitoneal sarcoma independently predicts overall survival (https://d oi.org/10.1245/s10434-021-10059-1). The complication burden tends to be greater in elderly, frail, and comorbid patients, which demonstrates the need to carefully identify these patients before surgery.

\section{Cumulative Burden of Postoperative Complications in Patients Undergoing Surgery for Primary Retroperitoneal Sarcoma}

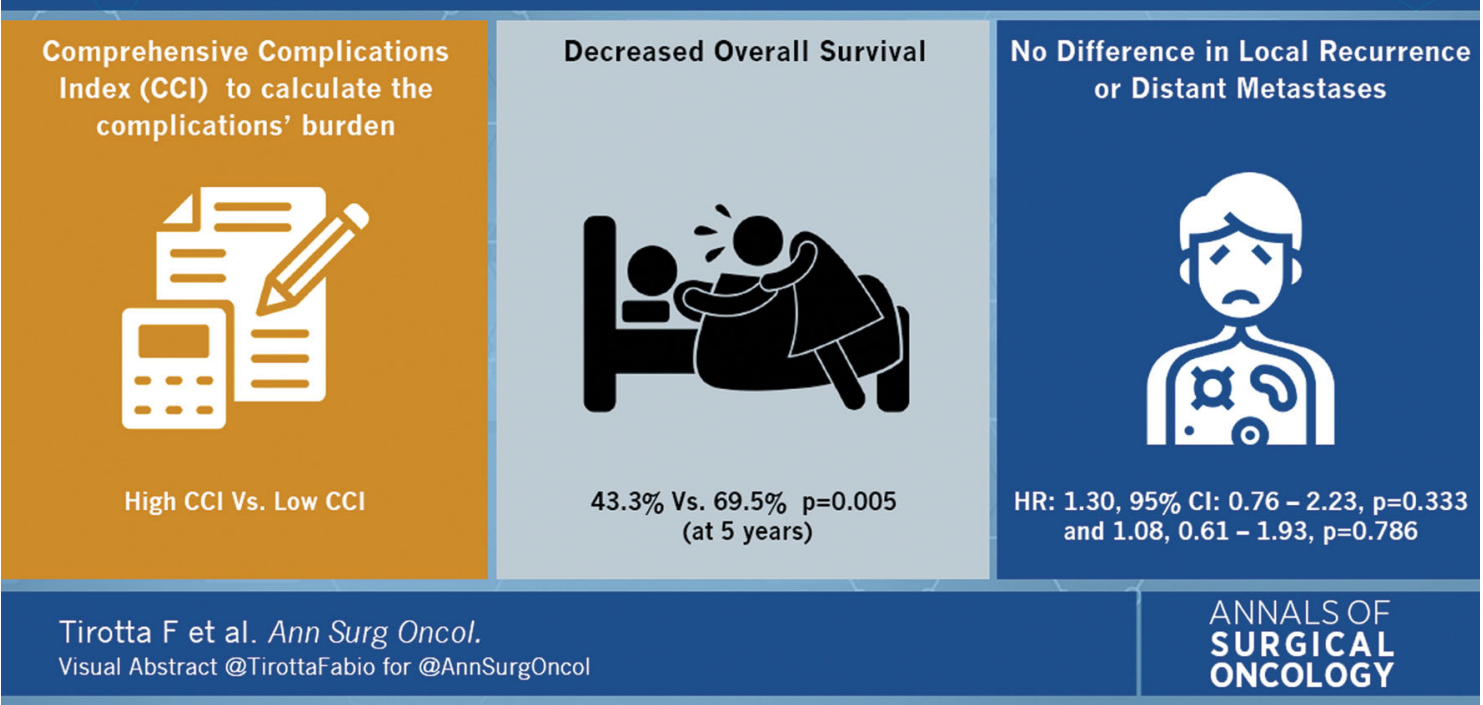

FUNDING None.

(C) Society of Surgical Oncology 2021

Published Online: 4 May 2021

F. Tirotta, MD

e-mail: fabio.tirotta.chirurgia@gmail.com
DISCLOSURES The authors declare that they have no conflict of interest.

Publisher's NoteSpringer Nature remains neutral with regard to jurisdictional claims in published maps and institutional affiliations. 